\title{
Preferences and Utilities for Treatment Attributes in Type 2 and Non-ambulatory Type 3 Spinal Muscular Atrophy in the United Kingdom
}

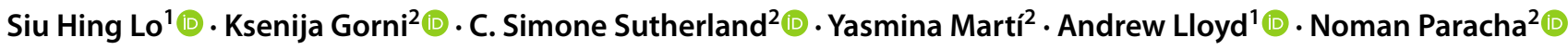

Accepted: 16 September 2021 / Published online: 18 October 2021

(c) The Author(s) 2021

\begin{abstract}
Background Spinal muscular atrophy (SMA) is a rare neuromuscular disease that affects motor neurons, resulting in progressive skeletal muscle weakness and atrophy.

Objective The aim was to understand the value patients with SMA and caregivers place on treatment attributes and to estimate health utilities for SMA treatment outcomes from a general public sample.

Methods Two discrete choice experiments were designed to elicit treatment preferences and health utilities, respectively. Patients with Type 2 and non-ambulatory Type 3 SMA, caregivers of patients with SMA and a general public sample in the UK completed the surveys. Patients and caregiver participants were recruited through patient associations. General public participants were recruited via a survey recruitment panel. Attributes included motor function, breathing function, treatment administration, treatment reactions, eyesight monitoring, contraception (patients only) and overall survival (general public only). Clustered conditional logit models were used to estimate treatment preferences, and marginal rates of substitution were used to estimate disutilities.

Results Adult patients $(n=84)$ were twice as likely to choose a treatment with improved (vs. stable) motor and breathing function and four to five times less likely to choose a treatment with deteriorated (vs. stable) motor and breathing function as a treatment outcome. Caregivers $(n=83)$ were three to nine times more likely to choose improved and two to four times less likely to choose deteriorated (vs. stable) motor and breathing function. Both patients and caregivers preferred oral over intrathecal treatment. Treatment reactions, eyesight monitoring or contraception had no significant effect on patient choices. Conversely, caregivers preferred avoidance of treatment reactions. General public data $(n=506)$ yielded disutilities for unable to sit $(-0.408)$, need for $>16 \mathrm{~h}$ daily mechanical breathing support $(-0.304)$ and intrathecal therapy $(-0.071)$.
\end{abstract} Conclusions Study results show the importance of motor and breathing function to patients and caregivers, and an oral treatment preference. Disutilities (decrements to utility) were substantial for SMA disease outcomes and care aspects.

Andrew Lloyd and Noman Paracha are senior authors.

Andrew Lloyd

andrew.1loyd@acasterlloyd.com

1 Acaster Lloyd Consulting Ltd, Lacon House, 84 Theobalds Road, WC1X 8NL London, UK

2 F. Hoffmann-La Roche Ltd, Basel, Switzerland

\section{Key Points for Decision Makers}

Caregivers and adult patients with Type 2 and nonambulatory Type 3 spinal muscular atrophy (SMA) value motor function, breathing function and oral administration in the context of SMA treatments.

Caregivers were more willing to make trade-offs to improve motor function and — to a lesser extent — breathing function, while adult patients focused more on avoiding deterioration in breathing function and motor function.

Disutilities generated from the UK population were substantial for SMA disease outcomes and care aspects. 


\section{Introduction}

Spinal muscular atrophy (SMA) is a severe autosomal recessive neuromuscular disorder [1-3]. SMA can be classified by clinical grades, based on age of onset and maximum motor function achieved [4]. Type 1 SMA has onset before 6 months of age. Type 2 SMA has onset after 6 months and before 18 months, whereas Type 3 SMA has onset after 18 months but before 18 years of age. Patients with Type 2 SMA are unable to stand or walk without support, whereas patients with Type 3 SMA can stand and walk, although these abilities are often lost as the disease progresses.

Nusinersen (SPINRAZA ${ }^{\circledR}$ ) was the first disease-modifying treatment (DMT) approved for patients with SMA [5]. It is administered via intrathecal injection (lumbar puncture) into the cerebrospinal fluid. After four loading doses within the first 2 months, patients receive a maintenance dose every 4 months. Intrathecal injections can be associated with treatment-related reactions such as headaches, vomiting and back pain $[6,7]$. New SMA treatments are emerging, including risdiplam (EVRYSDI ${ }^{\circledR}$ ) [8], which is given daily orally (or g-tube), and onasemnogene abeparvovec (ZOLGENSMA ${ }^{\circledR}$ ) [9], which is a gene therapy administered via an intravenous infusion.

A study of US patients with SMA examined patient and caregiver preferences for SMA treatment characteristics using a discrete choice experiment (DCE) [10]. Patients and caregivers placed high value on treatments that improve motor and breathing function, an indication across all ages, oral or one-time infusion, and minimal risk profiles. While the study provided insight into patient and caregiver preferences for SMA treatments, the US sample included caregivers of pediatric patients or patients with any SMA type and level of motor function. Further, the US study was not designed to understand preferences for avoiding the specific types of adverse events associated with nusinersen and risdiplam treatment.

The present study was designed to explore strength of preference among informal caregivers of patients and patients with Type 2 and non-ambulatory Type 3 SMA in the UK for different aspects of treatment for SMA, including treatment outcomes, mode of delivery and potential safety issues associated with nusinersen and risdiplam. Unlike the US SMA DCE study, this study was designed to understand treatment preferences among a more homogeneous group, caregivers of pediatric patients or patients with Type 2 and non-ambulatory Type 3 SMA. Second, the adverse event profiles of nusinersen and risdiplam were specifically used to inform attribute development in our study, allowing for patient preferences for relevant types of adverse events to be examined. In addition, the study was also designed to estimate SMA-related health utilities.
The study objective was to quantify caregiver and patient preferences for different attributes of SMA treatments for patients with Type 2 and non-ambulatory Type 3 SMA. The study survey was also adapted and presented to the general public to estimate health state utilities related to SMA.

\section{Methods}

\subsection{Study Design}

A three-part survey was developed for the patient/caregiver study: a screening questionnaire, a participant information sheet and informed consent, and the main survey. The main survey for adult patient and caregiver participants captured background demographic and clinical data, stated preference data (the DCE) and EQ-5D-5L data (for caregivers only) [11].

DCEs are a widely used method for eliciting patient treatment preferences $[12,13]$. A DCE survey describes hypothetical treatments in terms of their treatment characteristics ('attributes'). The attributes were based on known SMA treatments at the time the study was developed. Participants are asked to choose between pairs of hypothetical treatments, thus making trade-offs between different attributes. The relative importance of the different attributes can be estimated from the choices made by participants [14].

\subsection{Participant Recruitment and Study Procedures}

Survey participants were recruited through TreatSMA and SMA UK, patient associations based in the UK, via a closed Facebook group. Survey data were collected between November and December 2019. Survey completion time was measured to allow for data quality checks. If interested, participants were asked to complete a brief screening questionnaire to check whether they met the inclusion criteria:

1. Adult patient $\geq 18$ years) or parent/caregiver of a child ( $<18$ years) meeting inclusion the criteria below.

2. A (self-reported or proxy-reported) diagnosis of SMA.

3. Age of onset between 7 and 18 months (Type 2) or between 18 months and 17 years (Type 3).

4. Non-ambulatory, defined as being unable to walk for more than ten steps without a form of assistance.

All eligible participants then gave informed consent online using the informed consent text if they wished to proceed to the main survey. The online survey first provided descriptions of each treatment attribute, followed by 16 choice questions, each asking participants to choose between two treatments ('A' or 'B') (Fig. 1). Participants 
CHOICE SET 1 of 16

Please imagine that you are asked to choose a treatment for your child.

For each choice below please indicate whether you prefer treatment $\mathbf{A}$ or $\mathbf{B}$.

[PROGRAMMING INSTRUCTION: POP-UP BOXES FOR ALL TREATMENT ATTRIBUTES REPEATING ATTRIBUTE DESCRIPTIONS AS PRESENTED EARLIER]

\begin{tabular}{|l|}
\hline \\
\hline $\begin{array}{l}\text { Motor function } \\
\text { Level of change in motor function }\end{array}$ \\
\hline Breathing function \\
Change in breathing function \\
\hline $\begin{array}{l}\text { Administering the treatment } \\
\text { Treatment by injection in the lower back or by oral } \\
\text { liquid/gastric tube }\end{array}$ \\
\hline $\begin{array}{l}\text { Eyesight monitoring } \\
\text { The need for eyesight monitoring and risk of eye } \\
\text { changes and/or impact on vision }\end{array}$ \\
\hline $\begin{array}{l}\text { Treatment-related reaction } \\
\text { Treatments can cause reactions such as fever, } \\
\text { headache, vomiting and/or body pain }\end{array}$ \\
\hline
\end{tabular}

Which treatment do you prefer for your child?

\begin{tabular}{|c|}
\hline Hypothetical Treatment A \\
\hline $\begin{array}{l}\text { After } 12 \text { months, motor function will remain on the } \\
\text { current level of function }\end{array}$ \\
\hline After 12 months, breathing function will get better \\
\hline $\begin{array}{l}\text { Treatment is injected into the spine at the lower } \\
\text { back in hospital every four months, after an initial } \\
\text { set of four administrations }\end{array}$ \\
\hline $\begin{array}{l}\text { Eyesight monitoring before start of treatment and } \\
\text { during treatment if your child has symptoms }\end{array}$ \\
\hline $\begin{array}{c}\text { No fever, headache, vomiting and/or body pain due } \\
\text { to treatment }\end{array}$ \\
\hline
\end{tabular}

A

\begin{tabular}{|c|}
\hline Hypothetical Treatment B \\
\hline $\begin{array}{c}\text { After } 12 \text { months, motor function will have } \\
\text { deteriorated by one level of function }\end{array}$ \\
\hline After 12 months, breathing function will stay the \\
same
\end{tabular}

B

Fig. 1 Example choice question for caregivers' assessment of treatment preference

were also asked background socio-demographic and clinical questions, including the EQ-5D-5L (for caregivers only). After completion of the online survey, participants received a $£ 20$ e-voucher for an online retailer as remuneration for their time.

For the utility survey, a representative sample of the UK general public ( $n=500)$ were recruited by specialist recruitment panels. Potential participants were contacted by e-mail with a link to the survey and screened for eligibility. All participants were aged 18 years or over. Quota sampling was used in order to ensure geographical, gender and age representativeness based on UK census data. General population participants received the equivalent of approximately $£ 1$ in panel points as remuneration for their time.

\subsection{Ethical Review}

This study was reviewed and received exempt status determination by an independent review board, the Western Institutional Review Board (WIRB), on 6 June 2019 (submission number: \#1-1190961-1), prior to participant recruitment.

\subsection{Survey Development}

In the development of the stated preference survey, the first task involved the identification of appropriate attributes to describe the main treatments for SMA [15]. The aim was to identify the best attributes for describing treatment outcomes, mode of treatment administration and any safety issues. The survey was designed for patients with Type 2 and non-ambulatory Type 3 SMA and caregivers. The selection of attributes was driven by differences between nusinersen and risdiplam.

The literature regarding the nusinersen clinical trials was summarized. The Summary Product Characteristics from the European Medicines Agency for nusinersen and the risdiplam product profile were also reviewed. Literature regarding measures of motor function used in SMA was also included [16, 17]. Publications of trials and targeted searches were used to identify relevant literature.

The selection of attributes and survey design was guided by a need to keep the survey simple due to challenges recruiting large sample sizes in a rare disease. The attributes needed to be relevant for participants and to fairly reflect differences in the two treatments.

Different sources of information were used in the development of study attributes. D'Amico et al. (2011) presented a useful summary of the natural history of the main types of SMA, including the motor and respiratory burden that people can experience [3]. This is mirrored in the design of a clinical trial reported for nusinersen, where key endpoints related to motor milestones [18]. A large Australian study explored the expectations and insights of families affected by SMA [19]. This wide-ranging study provided many insights, but also underlined the very substantial effect of SMA on patients' quality of life, primarily due to the condition's impact on respiratory and motor function, but also psychosocial issues around the family and support from clinicians. 
Table 1 Motor function scale

\author{
Cannot sit \\ Can sit with some support (e.g., with back support or arm support) \\ Can sit independently for a few seconds \\ Can sit independently for a longer period of time but cannot stand \\ Can sit independently and stand with assistance, but cannot walk \\ Can sit independently and stand and walk with assistance \\ Can sit, stand and walk independently for a few steps (less than $10 \mathrm{~m}$ ) \\ In DCE choice sets only \\ Can sit, stand and walk independently over longer distances (more than $10 \mathrm{~m}$ )
}

Italic: short form used in remainder of report

$D C E$ discrete choice experiment; $\mathrm{m}$, meters
Lastly, the regulatory documents for nusinersen and the risdiplam profile helped to define attributes that focused on the mode of administration of the treatments and safety issues that may emerge from these treatments [5]. These safety issues related to the necessity to use contraceptives for birth control (for older adult patients) and also the possibility that the regulator could mandate regular ophthalmological testing for patients. The survey development work was undertaken prior to the regulatory approval of risdiplam.

Three SMA clinical experts, known for their involvement in SMA research and treatment, were interviewed to obtain feedback on the potential treatment attributes and physician experience since the introduction of nusinersen into clinical practice. Specifically, clinicians were asked to comment on the clinical relevance of potential attributes and overlap between attributes. For motor function, breathing function and treatment-related reactions, specific feedback on the clinical accuracy of the range presented in the attribute levels was also elicited. No specific feedback was sought on the levels for other treatment attributes, as these were based on known characteristics of nusinersen and likely characteristics of risdiplam. Following clinician feedback, five attributes (and a sixth attribute for the patient survey only) were included in the final DCE design. Other options for attributes to include in the survey (namely risk of infection and pulmonary problems other than breathing) were also discussed but considered of less or no relevance to patients with SMA.

A simple motor function scale was developed with input from clinical experts in order to characterize participants (Table 1). This was designed to capture a broad range of motor function for all levels of severity in patients with Type 2 and non-ambulatory Type 3 SMA. This was also used to describe the levels of this attribute in the DCE survey. A question was also developed to assess the level of breathing function as requiring mechanical support for $>16$ h daily, mechanical support for some of the day ( $<16 \mathrm{~h}$ daily) or ability to breathe without mechanical support.
The DCE survey questions were described in terms of five specific treatment attributes, which were in turn characterized by distinct levels.

1. Motor function (three levels): Motor function was described as having deteriorated by one level, remained stable at the current level or improved by one level after 12 months. Participants were shown the motor function scale on which they had previously identified the current level of motor function, with one additional level to represent improvement above the range experienced by patients with Type 2 and non-ambulatory Type 3 SMA without treatment (Table 1).

2. Breathing function (three levels): Described as worse, stable or better after 12 months.

3. Treatment administration (two levels): Treatment administered either orally via a liquid taken daily or an intrathecal injection every 4 months, after an initial set of four more frequent administrations.

4. Treatment reactions (three levels): Described as fever, headache, vomiting and body pain. This aimed to capture the presence and, if present, the duration of reactions (1-2 days every 4 months or 3-4 days every 4 months).

5. Eyesight monitoring (three levels): Described the requirement of eyesight monitoring and, if required, how often or under what conditions. This attribute was included because at the time of survey development it could have been a requirement for people receiving risdiplam on approval (risdiplam has since been approved by the Food and Drug Administration [FDA] and European Commission [EC] without this requirement) [8, 20]. The levels included no monitoring, monitoring before the start of treatment and if symptomatic, and monitoring before the start of treatment and twice yearly for the first 2 years of treatment.

6. Patient survey only — contraception (2 levels). Described the need for effective contraception while on treatment against no need for contraception. 
See the appendix in the electronic supplementary material for more information on the attributes and levels.

The attributes and levels were combined into choice sets using an orthogonal fractional factorial array that had been shifted by one level. The design was generated in SPSS version 25.0 using the orthoplan procedure. The survey consisted of 16 pairs of choice sets (Fig. 1). This resulted in two out of 16 choice sets where one choice was dominant, defined as having 'better' attribute levels for motor function, breathing function, treatment reactions, contraception, treatment administration (intrathecal injection considered 'worse') and eyesight monitoring (monitoring twice yearly was considered 'worse' than no monitoring or monitoring if symptoms). The survey was programmed to be completed online.

\subsection{Utility Survey}

The DCE survey was adapted for use with the general public. The main change involved the addition of an attribute that described overall life expectancy. Life expectancy was included to understand participants' willingness to trade overall length of life for gains in quality of life through the avoidance of SMA-related issues. This was designed as a corollary of the time trade-off method. Participants were informed that overall life expectancy would be reduced by between 0 and 12 years (four levels were specified: 0,4 , 8 or 12 years). The motor function attribute was changed to describe four absolute levels of motor function (being unable to sit, sitting, standing and walking independently for over $10 \mathrm{~m}$ ). Breathing function was changed to describe the need for mechanical breathing support for $>16 \mathrm{~h}$ of the day, $<16 \mathrm{~h}$ a day or not at all. The utility survey provided some background to the disease (without naming it).

After ethical approval, the survey was pilot tested with 42 members of the UK general public. The pilot results suggested no changes were required.

\subsection{Analysis}

After problems emerged in the first round of data collection, a minimum completion time of $175 \mathrm{~s}$ was set for the DCE choice questions, as the data showed that respondents who completed the survey under $175 \mathrm{~s}$ were more prone to give at least one illogical response to the dominated choice sets $(75 \%$ vs. $5 \%, P<0.001)$.

Background questions were analyzed using descriptive statistics. Choice data were analyzed using the conditional logit regression model with clustering on respondent level to account for repeated choices in Stata V16.0 [21, 22]. In comparison with a standard conditional logit model, a conditional logit model with clustering yields identical results for $\beta$ coefficients but adjusts standard errors to allow for intragroup correlation. The strength of preference associated with each attribute level was measured with respect to a reference level (i.e., all attributes were treated as categorical). Odds ratios were used to interpret the importance of each attribute. An alternative-specific constant was added to the model to account for any left bias.

Statistical analysis of the larger general public sample was conducted using the mixed-effect logit regression model in Stata V16.0 [22, 23]. The model was estimated using the maximum simulated likelihood approach. All attributes were initially specified as random coefficients, and choice scenarios were identified using a grouping variable. All attributes that did not show statistically significant standard deviations for the coefficients were included as fixed parameters in the final model. A higher-level grouping was specified at the level of respondent to account for multiple choice scenarios per respondent and to account for preference heterogeneity. In the model, the strength of preference associated with each attribute level was measured with respect to a reference level. An alternative-specific constant was added to the model to account for any left bias.

Marginal rates of substitution (MRS) were obtained by taking a ratio of the coefficients for two attributes: the life expectancy coefficient for 1 year and another treatment attribute. To calculate disutilities, we estimated the remaining life expectancy of the sample using the average age of men and women. Based on period life expectancy estimates for 2015-2017, the remaining life expectancy is 29.5 years for men aged 52 years and 38.0 years for women aged 46 years [24]. For simplicity, a single figure for remaining life expectancy was calculated based on the proportion of men (49\%) and women (51\%) in the sample:

$$
\begin{aligned}
0.49 & \times 29.5+0.51 \times 38 \\
= & 33.9 \text { years of remaining life expectancy }
\end{aligned}
$$

The MRS estimates were then used to estimate utilities for changes in treatment profiles. We have hypothesized that the MRS indicates the extent to which people are willing to trade length of life in order to avoid worse motor function, worse breathing function, a treatment administered via an intrathecal injection, treatment-related reactions, a requirement for eyesight monitoring and a need for contraception. This analysis is estimated at an aggregate level, and so the MRS is weighted in terms of predicted overall survival of the sample.

\section{Results}

\subsection{Sample Characteristics}

Table 2 summarizes the demographic and clinical characteristics of the pediatric patients under the care of a caregiver 
who took part in the study and adult patients who completed the survey themselves.

The vast majority of pediatric patients were proxyreported as having Type 2 SMA (92\%), with only a small proportion (8\%) reported as having Type 3 SMA. Pediatric patients were most commonly able to 'sit independently for longer' (81\%) or could 'stand with assistance' (16\%). Around half of pediatric patients (52\%) required some mechanical breathing support. Nearly two-thirds $(65 \%)$ of pediatric patients received nusinersen as a treatment for SMA. Almost all used a wheelchair (98\%) and a suction machine (95\%).

Similar to pediatric patients, most adult patients reported having Type 2 SMA (73\%), with a smaller proportion reporting Type 3 SMA (27\%). They could most commonly sit with some support (43\%), sit independently for a few seconds $(19 \%)$ or sit independently for longer $(21 \%)$. Over a quarter (27\%) required some mechanical breathing support. Most of adult patients received no treatment for their SMA (69\%), one $(1 \%)$ received nusinersen and $20 \%$ had undergone surgery. All (100\%) used a wheelchair, but few (12\%) used a suction machine.

Caregivers (mean age $=36$ years; $52 \%$ men) were typically employed full-time (59\%) or part-time (18\%) or were looking after their family/home (23\%). Over one-third (37\%) indicated they had reduced working hours, just under onethird (30\%) had changed jobs to be more flexible and almost a quarter (23\%) reported having stopped working as a result of caring. Most caregivers (84\%) had a child with SMA younger than 12 years. The most commonly reported chronic health conditions of caregivers were sleep problems (17\%) and hypertension (10\%). Caregivers reported relatively high health utility values overall, with worse scores reported for parents of more severely affected SMA patients.

The general public sample $(n=506)$ had a mean age of 48.7 years and $49 \%$ were male; $92 \%$ described themselves as white, $5 \%$ as Asian or British Asian and $4 \%$ as other ethnic groups. Geographic spread reflected the whole of the UK.

\subsection{Treatment Preferences: Adult Patients}

Of the 138 adult patients who were eligible and had consented to take part in the main survey, 103 completed the survey (the others dropped out between consent and completion). Of the 103 who completed the survey, 19 people (18\%) were removed from the sample because they had completed the DCE choice questions in under $3 \mathrm{~min}$. The analysis of the survey data shows that the motor function, breathing function, treatment administration attributes and all their associated attribute levels were statistically significant, independent predictors of treatment choice (Table 3). For adult patients, treatment reactions, eyesight monitoring and the need for contraception were not statistically significant predictors of choice.

Adult patients were 3.6 times less likely to prefer a treatment where motor function deteriorated and were 2.3 times more likely to prefer a treatment that improved motor function. Choices associated with deteriorating breathing function were 4.7 times less likely to be preferred than ones with stable breathing function. Treatments that improved breathing function were 2.4 times more likely to be preferred than treatments with stable breathing function. These results indicate that avoiding a deterioration in motor and breathing functions was more important to adult patients than an improvement in these functions.

Treatment administration also had a statistically significant influence on choice. Participants were 2.0 times more likely to choose a treatment taken orally on a daily basis than a treatment administered via an intrathecal injection every 4 months.

\subsection{Treatment Preferences: Caregivers}

The analysis shows that for caregivers all attributes (except eyesight monitoring) were relevant in their decision-making and were statistically significant, independent predictors of caregiver treatment choice in the survey (Table 4). The model results indicate that motor function was the most important attribute for caregivers. They were 8.9 times more likely to prefer a treatment that improved motor function by one level compared with stable function. Participants were 4.2 times less likely to prefer a treatment where motor function got worse.

Breathing function, treatment administration and treatment reaction had a smaller influence on choice. Treatments improving breathing function were 3.0 times more likely to be preferred than treatments with stable breathing function. Caregivers were also 2.9 times more likely to choose a treatment taken orally on a daily basis than a treatment administered via an intrathecal injection every 4 months. Treatments with no treatment-related reactions were 3.1 times more likely to be chosen than treatments with 3-4 days of fever, headache, vomiting and/or body pain. But treatment reactions that only lasted for 1-2 days were not significantly different to no risk of treatmentrelated reactions. The need for eyesight monitoring attribute was not a statistically significant predictor of caregiver treatment choices.

\subsection{Utility Weights: General Public}

All attributes and attribute levels were significant predictors of choice. The MRS data indicate that the impact of loss of motor function at the worst level is the same weight 
Table 2 Patient characteristics as described by caregivers (for pediatric patients) or by adult patient themselves and caregiver characteristics

\begin{tabular}{|c|c|c|}
\hline Patient characteristics & $\begin{array}{l}\text { Adult patient survey } \\
(N=84)\end{array}$ & $\begin{array}{l}\text { Caregiver survey } \\
\text { (proxy-reported) } \\
(N=83)\end{array}$ \\
\hline Age (years), mean (SD) & $33.9(11.5)$ & $8.4(3.4)$ \\
\hline Age at diagnosis (years), mean (SD) & $3.27(6.32)$ & $0.95(0.35)$ \\
\hline Age at first symptoms (years), mean (SD) & $1.43(1.34)$ & $0.78(0.35)$ \\
\hline Gender (male), $n(\%)$ & $23(27)$ & $75(90)$ \\
\hline \multicolumn{3}{|l|}{ Reported SMA type, $n(\%)$} \\
\hline Type 2 & $61(73)$ & $76(92)$ \\
\hline Type 3 & $23(27)$ & $7(8)$ \\
\hline \multicolumn{3}{|l|}{ Motor function, $n(\%)$} \\
\hline Cannot sit & $5(6)$ & $0(0)$ \\
\hline Can sit with some support & $36(43)$ & $3(4)$ \\
\hline Cannot sit independently for few seconds & $16(19)$ & $0(0)$ \\
\hline Can sit independently for longer & $18(21)$ & $67(81)$ \\
\hline Can stand with assistance & $6(7)$ & $13(16)$ \\
\hline Can walk with assistance & $3(4)$ & $0(0)$ \\
\hline Can walk independently for a few steps & $0(0)$ & $0(0)$ \\
\hline \multicolumn{3}{|l|}{ Breathing function, $n(\%)$} \\
\hline Mechanical support $>16 \mathrm{~h} /$ day & $1(1)$ & $0(0)$ \\
\hline Mechanical support for some of the day & $22(26)$ & $43(52)$ \\
\hline No mechanical support & $61(73)$ & $40(48)$ \\
\hline \multicolumn{3}{|l|}{ SMA treatment, $n(\%)$} \\
\hline Nusinersen (SPINRAZA ${ }^{\circledR}$ ) & $1(1)$ & $54(65)$ \\
\hline Surgery & $17(20)$ & $2(2)$ \\
\hline Other & $13(15)$ & $1(1)$ \\
\hline None & $58(69)$ & $29(34)$ \\
\hline \multicolumn{3}{|l|}{ Tools/equipment, $n(\%)$} \\
\hline Breathing machine/mechanical. ventilation & $30(36)$ & $45(54)$ \\
\hline Feeding tube & $9(11)$ & $0(0)$ \\
\hline Suction machine to help clear throat & $10(12)$ & $79(95)$ \\
\hline Walking frame & $1(1)$ & $39(47)$ \\
\hline Wheelchair & $84(100)$ & $81(98)$ \\
\hline Other & $22(26)$ & $1(1)$ \\
\hline \multicolumn{3}{|l|}{ Comorbidities (multicode), $n(\%)$} \\
\hline Any & $19(22)$ & $0(0)$ \\
\hline Musculoskeletal comorbidities & $10(12)$ & - \\
\hline Other comorbidities & $12(14)$ & - \\
\hline \multicolumn{3}{|l|}{ Caregiver EQ-5D-5L, mean (SD) } \\
\hline Can sit with some support $(n=3)$ & & $0.862(0.127)$ \\
\hline Can sit independently for longer $(n=67)$ & & $0.939(0.092)$ \\
\hline Can stand with assistance $(n=13)$ & & $0.964(0.074)$ \\
\hline
\end{tabular}

SMA spinal muscular atrophy as 13.8 years of life (assuming average life expectancy). We estimated that our overall sample had 33.9 years of life left on average, as calculated using the proportion of men and women in the sample. The utility loss associated with experiencing loss of motor function at the worst level ('cannot sit') is thus estimated as $13.8 / 33.9=-0.408$ compared with the reference category ('can sit, stand and walk independently for more than 10 m'; Table 5). Smaller losses in motor function had disutilities of -0.222 ("can sit but cannot stand') and -0.068 ('can sit, stand and walk with assistance'). The need for mechanical support was associated with a disutility of -0.304 ('mechanical support for some of the day') and -0.159 ('mechanical support for 
Table 3 Adult patient preference weights for SMA treatments from a conditional logit model with clustering

\begin{tabular}{|c|c|c|c|c|}
\hline Attributes and levels & $\beta$ coefficient & $\beta$ coefficient $95 \% \mathrm{CI}$ & Odds ratio & Odds ratio $95 \%$ CI \\
\hline \multicolumn{5}{|l|}{ Motor function-reference category: stable } \\
\hline Improved by one level & 0.851 & $0.557-1.145$ & 2.342 & $1.746-3.144$ \\
\hline Worse by one level & -1.294 & -1.539 to -1.050 & 0.274 & $0.215-0.350$ \\
\hline \multicolumn{5}{|l|}{ Breathing function-reference category: stable } \\
\hline Improved & 0.866 & $0.581-1.151$ & 2.378 & $1.788-3.162$ \\
\hline Worse & -1.539 & -1.879 to -1.200 & 0.215 & $0.153-0.301$ \\
\hline \multicolumn{5}{|c|}{ Treatment administration—reference category: oral liquid taken once daily at home } \\
\hline Injection into the spine in hospital every 4 months & -0.717 & -0.991 to -0.443 & 0.488 & $0.371-0.642$ \\
\hline \multicolumn{5}{|c|}{ Eyesight monitoring_reference category: no eye monitoring required } \\
\hline Monitoring during treatment if symptoms present & 0.117 & -0.083 to 0.318 & 1.125 & $0.920-1.374$ \\
\hline Monitoring twice a year for the first 2 years & 0.204 & -0.023 to 0.430 & 1.226 & $0.977-1.538$ \\
\hline \multicolumn{5}{|c|}{ Treatment reaction (fever, headache, vomiting and/or body pain)_reference category: no reaction } \\
\hline Reaction for $1-2$ days every 4 months & 0.018 & -0.219 to 0.254 & 1.018 & $0.803-1.289$ \\
\hline Reaction for 3-4 days every 4 months & 0.259 & -0.070 to 0.588 & 1.296 & $0.932-1.801$ \\
\hline \multicolumn{5}{|l|}{ Contraception } \\
\hline Must use effective contraception & -0.063 & -0.312 to 0.187 & 0.939 & $0.732-1.205$ \\
\hline
\end{tabular}

Figures in bold represent coefficients significant at $P<0.05$

Model statistics: Wald $\mathrm{Chi}^{2}(10)=235.37$; probability $>\mathrm{Chi}^{2}=<0.00$; pseudo $R^{2}=0.38$

CI confidence interval, SMA spinal muscular atrophy

Table 4 Caregiver preference weights for SMA treatments from a conditional logit model with clustering

\begin{tabular}{|c|c|c|c|c|}
\hline Attributes and levels & $\beta$ coefficient & $\beta$ coefficient $95 \% \mathrm{CI}$ & Odds ratio & Odds ratio $95 \% \mathrm{CI}$ \\
\hline \multicolumn{5}{|l|}{ Motor function-reference category: stable } \\
\hline Improved by one level & 2.188 & $1.730-2.647$ & 8.921 & $5.638-14.117$ \\
\hline Worse by one level & -1.431 & -1.830 to -1.031 & 0.239 & $0.160-0.357$ \\
\hline \multicolumn{5}{|l|}{ Breathing function-reference category: stable } \\
\hline Improved & 1.110 & $0.565-1.654$ & 3.034 & $1.760-5.230$ \\
\hline Worse & -0.847 & -1.225 to -0.470 & 0.429 & $0.294-0.625$ \\
\hline \multicolumn{5}{|c|}{ Treatment administration—reference category: oral liquid taken once daily at home } \\
\hline Injection into the spine in hospital every 4 months & -1.077 & -1.462 to -0.693 & 0.340 & $0.232-0.500$ \\
\hline \multicolumn{5}{|c|}{ Eyesight monitoring_reference category: no eye monitoring required } \\
\hline Monitoring during treatment if symptoms present & 0.089 & $-0.187-0.365$ & 1.093 & $0.830-1.440$ \\
\hline Monitoring twice a year for the first 2 years & -0.103 & $-0.375-0.168$ & 0.902 & $0.687-1.183$ \\
\hline \multicolumn{5}{|c|}{ Treatment reaction (fever, headache, vomiting and/or body pain)—reference category: no reaction } \\
\hline Reaction for $1-2$ days every 4 months & 0.131 & $-0.236-0.498$ & 1.140 & $0.790-1.645$ \\
\hline Reaction for 3-4 days every 4 months & -1.139 & -1.549 to -0.730 & 0.320 & $0.212-0.482$ \\
\hline
\end{tabular}

Figures in bold represent coefficients significant at $P<0.05$

Model statistics: Wald $\mathrm{Chi}^{2}(10)=306.18$; probability $>\mathrm{Chi}^{2}=<0.00$; pseudo $R^{2}=0.63$

CI confidence interval, SMA spinal muscular atrophy

$>16 \mathrm{~h}$ of the day') compared with the reference category ('can breathe without mechanical support'). Intrathecal injections had a disutility of -0.071 compared with a daily oral liquid. Table 5 also includes disutilities for treatment reactions, the need for eyesight monitoring and the need for contraception.

\section{Discussion}

This study describes a stated preference survey that was designed to examine adult patient and caregiver preferences for SMA treatments. The aim of the DCE is to evaluate the relative importance of treatment attributes to the participants 
Table 5 Estimated marginal utilities (expressed as a disutility) for differences in attribute levels

\begin{tabular}{|c|c|c|c|}
\hline & \multirow[t]{2}{*}{ Disutility } & \multicolumn{2}{|l|}{$95 \% \mathrm{CI}$} \\
\hline & & Lower bound & Upper bound \\
\hline \multicolumn{4}{|l|}{ Motor function } \\
\hline \multicolumn{4}{|l|}{ Can sit, stand and walk independently $(>10 \mathrm{~m})$} \\
\hline Can sit, stand and walk with assistance & -0.068 & -0.083 & -0.053 \\
\hline Can sit but cannot stand & -0.222 & -0.242 & -0.201 \\
\hline Cannot sit & -0.408 & -0.440 & -0.377 \\
\hline \multicolumn{4}{|l|}{ Breathing function } \\
\hline \multicolumn{4}{|l|}{ Can breathe without mechanical support } \\
\hline Mechanical support for some of the day $(<16 \mathrm{~h})$ & -0.159 & -0.174 & -0.143 \\
\hline Mechanical support for $>16 \mathrm{~h}$ of the day & -0.304 & -0.328 & -0.281 \\
\hline \multicolumn{4}{|l|}{ Treatment administration } \\
\hline \multicolumn{4}{|l|}{ Oral liquid taken once daily at home } \\
\hline Injection into spine in hospital every 4 months & -0.071 & -0.085 & -0.057 \\
\hline \multicolumn{4}{|l|}{ Treatment reaction } \\
\hline \multicolumn{4}{|l|}{ No treatment reactions } \\
\hline Reaction for $12 \mathrm{~h}$ every 4 months & -0.057 & -0.071 & -0.042 \\
\hline Reaction for $1-2$ days every 4 months & -0.060 & -0.078 & -0.042 \\
\hline Reaction for 3-4 days every 4 months & -0.087 & -0.103 & -0.071 \\
\hline \multicolumn{4}{|l|}{ Eyesight monitoring } \\
\hline \multicolumn{4}{|l|}{ No eye monitoring required } \\
\hline Monitoring if symptoms present & -0.024 & -0.036 & -0.012 \\
\hline Monitoring twice a year for the first 2 years & -0.023 & -0.037 & -0.009 \\
\hline \multicolumn{4}{|l|}{ Need for contraception } \\
\hline \multicolumn{4}{|l|}{ No requirement to use contraception } \\
\hline Must agree to use effective contraception & -0.012 & -0.021 & -0.002 \\
\hline
\end{tabular}

CI confidence interval; h, hours; $\mathrm{m}$, meters and to establish their willingness to trade between attributes. The survey focused on adult patients with Type 2 and non-ambulatory Type 3 SMA and the caregivers of pediatric patients. DMTs have only recently emerged for SMA. The survey has shown some differences in the treatment preferences of adults with SMA and caregivers of children with SMA.

Adult patients with SMA considered motor function and breathing function to be the most important attributes. Motor function and breathing function were both described in terms of improved, stable or declined function after 12 months. For motor function, the degree of change was described with respect to the motor function scale that was developed. They also preferred to avoid the need for intrathecal injections. Adult SMA patients' choices were not driven by avoidance of treatment reactions, eyesight monitoring or contraception. These attributes were not as important to adult patients.

For caregivers of pediatric patients with SMA, motor function was the most important treatment attribute, followed by respiratory function. The results indicate that caregivers placed relatively more value on improvements than avoiding deterioration in motor and respiratory function. Caregivers also preferred to avoid treatment administration via intrathecal injections and preferred to avoid treatment reactions that lasted 3-4 days, but were not concerned about reactions that only last 1-2 days. Eyesight monitoring was not a significant concern.

Adult patients and caregivers alike valued improvements in motor function and breathing function, but there were some interesting differences. Adult patients placed relatively more value on avoiding deterioration in motor function and breathing function, which contrasts with caregivers of pediatric patients, who placed more value on gaining improvements in motor and breathing function. Adult patients may place relatively greater value on stabilization, as it can enable them to maintain autonomy and could therefore be perceived as a marker of treatment success among adults [25]. Caregivers preferred to avoid longer-lasting treatment reactions, whereas for adult patients, this was not a significant driver of their choices, possibly suggesting that adults with SMA are willing to tolerate these. These differences may be quite important for physicians and decision-makers to be aware of, namely that adult patients display a quite significant loss aversion which may reflect their experience with the disease over their lifetime and a desire to maintain 
independence. However, it is also possible that these differences in preferences reflect differences in the samples in terms of patient characteristics.

The general public data also provide additional insights into societal perspectives on SMA. The disutilities show the value that the public placed on avoiding being restricted to sitting only and being reliant on mechanical ventilation. These outcomes in SMA are associated with very substantial declines in utility. Other outcomes were also related to small (but statistically significant) changes in utility too. Intrathecal injections had a significant disutility $(-0.071)$ as did treatment-related reactions $(-0.057$ to -0.087$)$. These DCE-derived disutilities are a departure from the use of EQ-5D data collection, but in a rare disease like SMA, it would be difficult to estimate the disutilities of these outcomes using EQ-5D. Further work is needed to validate the changes in utility using this method.

These data have several applications to support decisionmaking. In the advent of new DMTs becoming available for the SMA community, families affected by SMA and physicians who treat patients with SMA have a choice of treatment. These data suggest that the choice should not be guided by clinical effectiveness only but be guided by many factors. For example, both patient and caregiver groups valued the avoidance of intrathecal injection and caregivers preferred for their children to avoid longer-lasting treatment reactions.

The caregiver, patient and general public preference data can also be used to inform national-level decision-makers who are concerned with understanding the value of improvements in treatment. The value of moving to an oral therapy can be benchmarked against the value of improvements in motor and breathing function. The DCE methodology allows us to understand the extent to which patients and caregivers are willing to trade one attribute against another. The nonsignificant attributes such as need for contraception or eyesight monitoring indicate that these are issues that patients and caregivers are willing to accept, and were not considered sufficiently important to influence their choices.

This study has some limitations that should be considered. First, the study results might not be (fully) generalizable to patients with Type 1 or ambulatory SMA. However, a recent preference study among US-based patients with Type 1-4 SMA and caregivers reported preferences that were broadly consistent with the present study results [10]. The US study had a different but overlapping set of attributes and attribute levels; so, while consistent, the results of the two studies cannot be directly compared. Despite the relative homogeneity of the sample, there were differences in physical function between caregivers of pediatric patients and patients in the study sample. Due to the small overall study sample and distribution of the sample across levels of motor function, it was not possible to examine if preferences varied between subgroups with different levels of function. The sample in the present study also included a much larger proportion of caregivers of pediatric patients and patients with Type 2 SMA than Type 3 SMA. Results may therefore have been skewed towards preferences of those with Type 2 SMA, even though those with non-ambulatory Type 3 SMA were also included. Secondly, the present study included a limited number of treatment attributes and attribute levels. The adult patient sample included a very small number of patients who reported functioning at the worst level of the motor function scale used in this study. While it was possible for these patients to imagine a worse level of function based on their personal experience and knowledge, the study did not provide explicit guidance on what this would entail. The study also did not consider some aspects of SMA disease morbidity, especially those impacting long-term health-related quality of life and prognosis among adults with SMA, who may have bulbar weakness resulting in difficulties with swallowing and feeding that could lead to aspiration pneumonia, excessive fatigue, osteoporosis, kidney stones, acidosis and weight management, sexual health and hormonal issues $[25$, $26]$. The study also did not actively involve patients in the development of attributes and survey pilot testing to ensure patient relevance and understanding of the DCE survey, or whether patients believed the hypothetical choice scenarios were plausible and how this affected their choices. For example, it is unknown if and how presenting hypothetical treatment options that are administered via a daily oral liquid and associated with treatment reactions occurring every 4 months affected participants' choices.

The survey recruited caregivers and patients via a patient association using a closed Facebook group for patients with SMA. We could not verify people's identity and caregiver or patient status, and it is possible that patients in the Facebook group were not representative of the wider SMA population. Furthermore, in a survey, we cannot be sure that people read all of the information they receive. Therefore, we used survey completion time to remove respondents who had rushed completing the survey in the adult patient sample. The same quality check could not be applied to the caregiver data, as completion time was not recorded for that survey.

This survey did not make any reference to treatment via gene therapy, which is now available for a minority of patients with Type 2 and 3 SMA [9, 27], as at the time the study was designed it was not deemed a relevant treatment option for these patients. In principle, the results regarding the value of improvements in motor and respiratory function could be applied to a gene therapy as well. However, important aspects of a gene therapy such as the onceonly administration, any risks associated with intravenous 
treatment administration, and the long-term efficacy of the treatment were not incorporated into the DCE survey.

While the dropout rate during survey completion was low among caregivers, a substantial proportion of adult patients started the survey but did not finish. However, a closer examination of the patient characteristics showed no statistically significant differences between those who completed the survey and those who dropped out.

\section{Conclusion}

This stated preference survey shows the value that caregivers and adult patients place on motor function, breathing function and oral administration in the context of SMA treatments. While caregivers were more willing to make trade-offs to improve motor function and-to a lesser extent-breathing function, adult patients focused more on avoiding deterioration in breathing function and motor function. Caregivers also valued avoiding longer-lasting treatment-related reactions, whereas for adult patients, this was not a significant issue. Eyesight monitoring requirements and the need for contraception did not significantly affect caregiver and patient treatment choices.

Supplementary Information The online version contains supplementary material available at https://doi.org/10.1007/s40273-021-01092-9.

Acknowledgements We would like to thank study participants for their help, by taking part in the survey, and TreatSMA, SMA UK and Jack Robinson for their help recruiting study participants. In addition, we thank and Gautam P. Ambegaonkar, Marianne Gerber, Francesco Muntoni and Kayal Vijayakumar for their clinical insights and Dylan Trundell for help with study design. The authors thank Helena Bailes, $\mathrm{PhD}$, of MediTech Media, UK, for providing medical writing support, which was funded by F. Hoffmann-La Roche Basel Ltd, Switzerland, in accordance with Good Publication Practice (GPP3) guidelines (http:// www.ismpp.org/gpp3).

\section{Declarations}

Funding This study was sponsored by F. Hoffmann-La Roche Ltd, Basel, Switzerland.

Disclosure statement This article is published in a special edition journal supplement wholly funded by F. Hoffmann-La Roche Ltd.

Conflict of interest SHL and AL are employees of Acaster Lloyd Consulting Ltd, who were commissioned by F. Hoffmann-La Roche Ltd to conduct this study and therefore received a consulting fee to conduct the study presented in the article. CSS, YM and KG are employees of F. Hoffmann-La Roche Ltd. During the preparation of the manuscript, NP was employed by F. Hoffmann-La Roche. NP and KG are shareholders of F. Hoffmann-La Roche. CSS is a shareholder of Roche Holding AG.
Availability of data and material The authors adhere to General Data Protection Regulation (GDPR) and UK Data Protection requirements, so will not make individual de-identified participant data available, as no consent to share the data publicly has been obtained; however, the study protocol will be made available upon request to the corresponding author.

Code availability Not available.

Author contributions SHL and AL designed the study. SHL analyzed the data. SHL and AL interpreted the data and drafted the manuscript. $\mathrm{NP}$ and KG contributed to the design of the study, interpreted the data and revised the draft critically for important intellectual content. CSS and YM interpreted the data and revised the draft critically for important intellectual content. All authors approved the final version of the manuscript and agree to be accountable for all aspects of the work in ensuring that questions related to the accuracy or integrity of any part of the work are appropriately investigated and resolved.

Ethics approval This study was reviewed and received exempt status determination by an independent review board, the Western Institutional Review Board (WIRB), on 6 June 2019 (submission number: \#1-1190961-1), prior to participant recruitment.

Consent to participate Freely given, informed consent to participate in the study was obtained from participants prior to participation in the study.

Consent for publication Freely given, informed consent for the scientific publication of the results of this research was obtained from participants prior to participation in the study.

Open Access This article is licensed under a Creative Commons Attribution-NonCommercial 4.0 International License, which permits any non-commercial use, sharing, adaptation, distribution and reproduction in any medium or format, as long as you give appropriate credit to the original author(s) and the source, provide a link to the Creative Commons licence, and indicate if changes were made. The images or other third party material in this article are included in the article's Creative Commons licence, unless indicated otherwise in a credit line to the material. If material is not included in the article's Creative Commons licence and your intended use is not permitted by statutory regulation or exceeds the permitted use, you will need to obtain permission directly from the copyright holder. To view a copy of this licence, visit http://creativecommons.org/licenses/by-nc/4.0/.

\section{References}

1. Feldkotter M, Schwarzer V, Wirth R, Wienker TF, Wirth B. Quantitative analyses of SMN1 and SMN2 based on real-time lightCycler PCR: fast and highly reliable carrier testing and prediction of severity of spinal muscular atrophy. Am J Hum Genet. 2002;70(2):358-68.

2. Lunn MR, Wang CH. Spinal muscular atrophy. Lancet (London, England). 2008;371(9630):2120-33. https://doi.org/10.1016/ S0140-6736(08)60921-6.

3. D'Amico A, Mercuri E, Tiziano FD, Bertini E. Spinal muscular atrophy. Orphanet J Rare Dis. 2011;6:71.

4. Russman BS. Spinal muscular atrophy: clinical classification and disease heterogeneity. J Child Neurol. 2007;22(8):946-51.

5. Food and Drug Administration. Approval Package for Spinraza Injection, $2.4 \mathrm{mg} / \mathrm{mL}$. 2016. https://www.accessdata.fda. 
gov/drugsatfda_docs/nda/2016/209531Orig1s000Approv.pdf. Accessed Oct 2021.

6. Claborn MK, Stevens DL, Walker CK, Gildon BL. Nusinersen: a treatment for spinal muscular atrophy. Ann Pharmacother. 2019;53(1):61-9. https://doi.org/10.1177/1060028018789956.

7. European Medicines Agency. Spinraza: EPAR — Product Information. 2019. https://www.ema.europa.eu/en/documents/productinformation/spinraza-epar-product-information_en.pdf. Accessed Oct 2021.

8. Food and Drug Administration. EVRYSDI ${ }^{(\mathrm{R})}$ Highlights of prescribing information. 2020. https://www.accessdata.fda.gov/drugs atfda_docs/label/2020/213535s000lbl.pdf. Accessed Oct 2021.

9. AveXis Inc. ZOLGENSMA (onasemnogene abeparvovec-xioi) [package insert]. Bannockburn, IL. 2019. https://www.fda.gov/ media/126109/download. Accessed Oct 2021.

10. Monnette A, Chen E, Hong D, Bazzano A, Dixon S, Arnold WD, et al. Treatment preference among patients with spinal muscular atrophy (SMA): a discrete choice experiment. Orphanet J Rare Dis. 2021;16(1):36. https://doi.org/10.1186/s13023-020-01667-3.

11. EuroQol Research Foundation. EQ-5D-5L. 2021. https://euroqol. org/eq-5d-instruments/eq-5d-51-about/. Accessed Oct 2021.

12. Johnson F, Lancsar E, Marshall D, Kilambi V, Mühlbacher A, Regier D, et al. Constructing experimental designs for discretechoice experiments: report of the ISPOR conjoint analysis experimental design good research practices task force. Value Health. 2013;16(1):3-13. https://doi.org/10.1016/j.jval.2012.08.2223.

13. Hauber A, Marcos González J, Groothuis-Oudshoorn C, Prior T, Marshall D, Cunningham C, et al. Statistical methods for the analysis of discrete choice experiments: a report of the ISPOR conjoint analysis good research practices task force. Value Health. 2016;19(4):300-15. https://doi.org/10.1016/j.jval.2016.04.004.

14. De Bekker-Grob E, Ryan M, Gerard K. Discrete choice experiments in health economics: a review of the literature. Health Econ. 2012;21(2):145-72. https://doi.org/10.1002/hec.1697.

15. Bridges JFP, Hauber AB, Marshall D, Lloyd A, Prosser LA, Regier DA, et al. Conjoint analysis applications in healtha checklist: a report of the ISPOR good research practices for conjoint analysis task force. Value Health. 2011;14(4):403-13. https://doi.org/10.1016/j.jval.2010.11.013.

16. WHO Multicentre Growth Reference Study Group. WHO Motor Development Study: windows of achievement for six gross motor development milestones. Acta Paediatr Suppl. 2006;450:86-95. https://doi.org/10.1111/j.1651-2227.2006.tb02379.x.

17. Berard C, Payan C, Hodgkinson I, Fermanian J. A motor function measure for neuromuscular diseases. Construction and validation study. Neuromuscul Disord. 2005;15(7):463-70.

18. Mercuri E, Darras BT, Chiriboga CA, Day JW, Campbell C, Connolly AM, et al. Nusinersen versus sham control in later-onset spinal muscular atrophy. N Engl J Med. 2018;378:625-35.

19. Centre for Community-Driven Research (CCDR), SMA Australia, Muscular Dystrophy New South Wales, Muscular Dystrophy Western Australia, Muscular Dystrophy Queensland, Muscular Dystrophy Australia, et al. Patient Experience, Expectations and Knowledge: Spinal Muscular Atrophy Australian Study. PEEK. 2017.

20. Roche. Evrysdi (risdiplam) summary of product characteristics. 2021. https://www.ema.europa.eu/en/documents/product-infor mation/evrysdi-epar-product-information_en.pdf. Accessed Oct 2021.

21. McFadden D. Conditional logit analysis of qualitative choice behavior. In: Zarembka P, editor. In frontiers in econometrics. New York: Academic Press; 1974.

22. StataCorp, . Stata statistical software: release 16. College Station: StataCorp LLC; 2019.

23. Hole AR. Fitting mixed logit models by using maximum simulated likelihood. Stand Genomic Sci. 2007;7(3):388-401.

24. Office for National Statistics. National life tables, UK: 2014 to 2016. 2017. https://www.ons.gov.uk/peoplepopulationandc ommunity/birthsdeathsandmarriages/lifeexpectancies/bulletins/ nationallifetablesunitedkingdom/2014to2016. Accessed Oct 2021.

25. Walter M, Chiriboga C, Duong T, Goemans N, Mayhew A, Laëtitia $\mathrm{O}$, et al. Improving care and empowering adults living with SMA: a call to action in the new treatment era. J Neuromuscul Dis. 2021. https://doi.org/10.3233/JND-200611.

26. Wan HWY, Carey KA, D'Silva A, Vucic S, Kiernan MC, Kasparian NA, et al. Health, wellbeing and lived experiences of adults with SMA: a scoping systematic review. Orphanet J Rare Dis. 2020;15(1):70. https://doi.org/10.1186/s13023-020-1339-3.

27. EMA. ZOLGENSMA ${ }^{\circledR}$ (onasemnogene abeparvovec-xioi) EMA prescribing information. 2020. https://www.ema.europa.eu/en/ documents/product-information/zolgensma-epar-product-infor mation_en.pdf. Accessed Oct 2021. 